\title{
The Application of Distributional Henstock-Kurzweil Integral on Third-order Three-Point Nonlinear Boundary-Value Problems
}

\author{
Dakai Zhou ${ }^{1}$, Guoju Ye ${ }^{1}$, Wei Liu ${ }^{1} \&$ Bing Liang ${ }^{1}$ \\ ${ }^{1}$ College of Science, Hohai University, Nanjing 210098, P.R. China \\ Correspondence: Dakai Zhou ,College of Science,Hohai University, Nanjing 210098, P.R.China. \\ E-mail: zhoudakai24@163.com
}

Received: October 5, 2015 Accepted: November 4, 2015 Online Published: November 24, 2015

doi:10.5539/jmr.v7n4p150 URL: http://dx.doi.org/10.5539/jmr.v7n4p150

\begin{abstract}
We consider a type of form such as $u^{\prime \prime \prime}=-f$ with three-point nonlinear boundary-value problems (NBVPs). We verified the existence of solutions of the (NBVPs) when $f$ is distributional Henstock-Kurzweil integral but not Henstock-Kurzweil integral.We use the distribution derivative and fixed point theorem to deal with the problem. The results obtained generalize the known results. For this reason, it is conducive to the further study of NBVPS.
\end{abstract}

Keywords: distributional Henstock-Kurzweil integral, distributional derivative, nonlinear boundary value problems, Fubini theorem, fixed point theorem

\section{Introduction}

This paper is devoted to study the extended existence of solutions of the specific given third-order differential equation with the boundary conditions

$$
\left\{\begin{array}{l}
u^{\prime \prime \prime}(t)=-f(t, u(t)), \quad t \in[0,1], \\
u(0)-p u^{\prime}(0)=0, \quad u^{\prime}(\xi)=0, \quad u(1)=0,
\end{array}\right.
$$

where $u^{\prime \prime \prime}, u^{\prime}$ stand for the distributional derivative of the function $u \in C^{2}[0,1], C^{2}[0,1]$ denotes the space where the functions $u^{\prime \prime}:[0,1] \rightarrow \mathbb{R}$ are continuous, $f$ is a distribution (generalized function), $p \in \mathbb{R}$ and $\xi \in[0,1]$. The spaces $C[0,1]$ is considered with the uniform norm $\|\cdot\|_{\infty}, C^{2}[0,1]$ is considered with the norm $\|u\|_{C^{2}}=\|u\|_{\infty}+\left\|u^{\prime}\right\|_{\infty}+\left\|u^{\prime \prime}\right\|_{\infty}$.

In 1989, Lee indicates that if $F$ is a continuous function and pointwise differentiable almost everywhere on [a,b], then $F$ is ACG*(generalized absolutely continuous). A primitive $F$ of the Henstock-Kurzweil-integrable function $f$ is ACG* (see[J. Kurzweil (1999); Š. Schwabik, \& Guoju Ye, (2005); E. Talvila. (1999-2000)]). However,let us consider the function:

$$
F(t)=\sum_{n=1}^{\infty} \frac{\sin n^{2} t}{n^{2}}, \quad t \in \mathbb{R}
$$

As we all know, $F(t)$ is continuous but not differentiable almost everywhere.It means $F$ is not ACG*. Nevertheless, $F(t)$ has distributional derivative. That is to say, there exist a function $f$ satisfy $F^{\prime}=f$ ("'" denotes the distributional derivative) therefore $f$ is distributional Henstock-Kurzweil integrable, but $f$ is not Henstock-Kurzweil integrable.Moreover,T. S. Chew and F. Flordeliza, in [ T. S. Chew, \& F. Flordeliza. (1991)], generalized the classic Carathéodory's existence theorem on the Cauchy problem $u^{\prime}=f(t, u)$ with $u(0)=0$. Since the BVPs has been studied by using ordinary derivative or approximate derivative in [D. Anderson,T. Anderson, \& M. Kleber. (2006); P. Minghe, \& S. K. Chang. (2007)], and from [D. Bugajewska. (1998); P. S. Bullen, \& D. N. Sarkhel. (1987); Xueyuan Zhou, \& Guoju Ye. (2012); Bing Liang, \& Guoju Ye. (2015, May 1)] we get the motivate to study the third-order nonlinear boundary value problems. we use the distributional derivatives to discuss (1) in a general form. Naturally, we can obtain the existence of solutions of the equation(1) under weaker conditions. This makes the integrand of the third-order differential equations more extensive.

The remainder of this paper is organized as follows. In Section 2, we introduce the definitions and the properties of the distributional Henstock-Kurzweil integral. In section 3, we apply the Schauder's fixed point theorem to verify the existence of the NBVP (1). In section 4, An example is provided to illustrate the effectiveness of the proposed theorem 3.1 in this paper.

\section{The Distributional Henstock-Kurzweil Integral}

In this section, the definition of the distributional Henstock-Kurzweil integral and their main properties needed in this paper are presented. 
Define the space of test functions

$$
\mathcal{D}=\left\{\phi: \mathbb{R} \rightarrow \mathbb{R} \mid \phi \text { has compact support in } \mathbb{R} \text { and } \phi \in C^{\infty}\right\},
$$

where the compact support of $\phi$ denoted by $\operatorname{supp}(\phi)=\overline{\{x \in \mathbb{R}: \phi(x) \neq 0\}}$. A sequence $\left\{\phi_{n}\right\} \subset \mathcal{D}$ converges to $\phi \in \mathcal{D}$ means that a compact set $K$ such that all $\phi_{n}$ have support in $K$ and for each integer $m \geq 0$, the sequence of derivatives $\phi_{n}^{(m)}$ converges to $\phi^{(m)}$ uniformly. $\mathcal{D}^{\prime}$ denotes the dual space of $\mathcal{D}$. It indicate that $\mathcal{D}^{\prime}$ is the set of all functional $f: \mathcal{D} \rightarrow \mathbb{R}$, denoted $f(\phi)=\langle f, \phi\rangle, \phi \in \mathcal{D}$.

We define the distributional derivative $f^{\prime}$ of $f$ to be a distribution satisfying $\left\langle f^{\prime}, \phi\right\rangle=-\left\langle f, \phi^{\prime}\right\rangle$, where $\phi \in \mathcal{D}$ and $\phi^{\prime}$ is the ordinary derivative of $\phi$. With this definition, it is easy to get that every distribution is infinite differentiability in the distributional derivative sense.

Let $(a, b)$ be an open interval in $\mathbb{R}$. We define

$$
\mathcal{D}((a, b))=\left\{\phi:(a, b) \rightarrow \mathbb{R} \mid \phi \in C_{c}^{\infty} \text { and } \phi \text { has compact support in }(a, b)\right\} .
$$

The dual space to $\mathcal{D}((a, b))$ is denoted by $\mathcal{D}^{\prime}((a, b))$.

Denote $C[a, b]$ the space of continuous functions on $[a, b]$ and let $B_{C}=\{F \in C[a, b] \mid F(a)=0\}$. We can obtain $B_{C}$ is a Banach space with the uniform norm

$$
\|F\|_{\infty}=\max _{t \in[a, b]}|F(t)| .
$$

Next, we give an introduction about the definition and properties of the $D_{H K}$-integral.

Definition 2.1 A distribution $f$ is said to be distributionally Henstock-Kurzweil integrable on $[a, b]$ or briefly $D_{H^{-}}$ integrable if $f$ is the distributional derivative of a continuous function $F \in B_{C}$.

For succinctness, we will refer to " $\left(D_{H K}\right) \int$ " as simply " $\int$ ". Denote $D_{H K}=\left\{f \in \mathcal{D}^{\prime}((a, b)) \mid f=F^{\prime}\right.$ for some $\left.F \in B_{C}\right\}$. In this case, if $f \in D_{H K}$, then $\left(D_{H K}\right) \int_{a}^{b} f=F(b)$, and for all $\phi \in \mathcal{D}((a, b))$

$$
\langle f, \phi\rangle=\left\langle F^{\prime}, \phi\right\rangle=-\left\langle F, \phi^{\prime}\right\rangle=-\int_{a}^{b} F \phi^{\prime}, \text { where } F \in B_{C} .
$$

Definition 2.2 Let $Q=[a, b] \times[c, d]$ in $\mathbb{R}^{2} \widetilde{B}_{C}=\{H \in C(Q): H(a, y)=H(x, c)=0$, for every $x \in[a, b], y \in[c, d]\}$, we denote " $\partial_{1}$ " and " $\partial_{2}$ " the distributional derivatives with respect to $x$ and $y$ respectively. Define

$$
D_{H K}(Q)=\left\{f \in \mathcal{D}^{\prime}(Q): f=\partial F, F \in \widetilde{B}_{C}\right\} .
$$

We can know that if $f \in D_{H K}(Q)$, then the corresponding continuous function $F\left(F \in \widetilde{B}_{C}\right.$ and $f=\partial F$ )is unique.

Definition $2.3 f \in D_{H K}(Q), x \in[a, b], y \in[c, d]$. We define

$$
\begin{array}{lll}
\int_{a}^{x} f(\zeta, \cdot) d \zeta=\partial_{2} F_{f}(x, \cdot) & \text { in } & \mathcal{D}^{\prime}((c, d)) \\
\int_{c}^{y} f(\cdot, \eta) d \eta=\partial_{1} F_{f}(\cdot, y) & \text { in } & \mathcal{D}^{\prime}((a, b))
\end{array}
$$

It is clear that

$$
\int_{a}^{x} f(s, \cdot) d s \in D_{H K}((c, d)), \int_{c}^{y} f(\cdot, t) d t \in D_{H K}((a, b)) .
$$

Let us introduce some basic properties of the distributional Henstock-Kurzweil integral needed later.

Lemma 2.1 (Fundamental Theorem of Calculus, [E. Talvila. (2008), Theorem 4] ).

(a) Let $f \in D_{H K}$, and define $F(t)=\int_{a}^{t} f$. Then $F \in B_{C}$ and $F^{\prime}=f$.

(b) Let $F \in C[a, b]$. Then $\int_{a}^{t} F^{\prime}=F(t)-F(a)$ for all $t \in[a, b]$.

We define the Alexiewicz, norm with

$$
\|f\|=\sup _{0 \leq t \leq 1}\left|\int_{0}^{t} f(s) d s\right|=\|F\|_{\infty}, f \in D_{H K} \text { and } F \in B_{C} .
$$


A sequence $\left\{f_{n}\right\} \subset D_{H K}$ converges strongly to $f \in D_{H K}$, if $\left\|f_{n}-f\right\| \rightarrow 0$ as $n \rightarrow \infty$. According to those, the following result holds.

Lemma 2.2 (Theorem 2, [E. Talvila. (2008)]). $D_{H K}$ is a Banach space with the Alexiewicz norm.

Lemma 2.3 (Definition 6, Integration by parts), [E. Talvila.(2008)]. Let $f \in D_{H K}$, and $g$ is a function of bounded variation. Define $f g=D H$, where $H(t)=F(t) g(t)-\int_{a}^{t} F d g$. Then $f g \in D_{H K}$ and

$$
\left(D_{H K}\right) \int_{a}^{b} f g=F(b) g(b)-\left(D_{H K}\right) \int_{a}^{b} F d g .
$$

Now we introduce a partial ordering on $D_{H K}$ : for $f, g \in D_{H K}$, we say that $f \geq g$ (or $g \leq f$ ) if and only if $f-g$ is a positive measure on $[a, b]$. By the definition, if $f, g \in D_{H K}$, then $\int_{I} f \geq \int_{I} g$ for every $I=[c, d] \subset[a, b]$, whenever $f \geq g$. See [D. D. Ang, K. Schmitt, \& L. K. Vy. (1997)] for details.

From the above mentioned, It is easy to see that the following result hold.

Lemma 2.4 (Corollary 5 Dominated convergence theorem for the $D_{H K}$-integral,[D. D. Ang, K. Schmitt, \& L. K. Vy. (1997)]) Let $\left\{f_{n}\right\}_{n=0}^{\infty}$ be a sequence in $D_{H K}$ such that $f_{n} \rightarrow f$ as $n \rightarrow \infty$ in $\mathcal{D}^{\prime}$. Suppose there exist $f_{-}, f_{+} \in D_{H K}$ satisfying $f_{-} \leq f_{n} \leq f_{+}$, for $\forall n \in \mathbb{N}$. Then $f \in D_{H K}$ and $\lim _{n \rightarrow \infty} \int_{a}^{b} f_{n}=\int_{a}^{b} f$.

Lemma 2.5 (Theorem 4, Fubini theorem, [D. D. Ang, K. Schmitt, \& L. K. Vy. (1997)] ) For all $f \in D_{H K}(Q)$, we have

$$
\int_{Q} f=\int_{a}^{b}\left(\int_{c}^{d} f(\cdot, \eta) d \eta\right)=\int_{c}^{d}\left(\int_{a}^{b} f(\zeta, \cdot) d \zeta\right) .
$$

The next statement is modified from [E. Talvila. (1999-2000)] and [E. Talvila. (2008)].

Lemma 2.6 Let $f \in D_{H K}$ and $\left\{f_{n}\right\}_{n=0}^{\infty}$ be a sequence in $D_{H K}$ such that $f_{n} \rightarrow f$ as $n \rightarrow \infty$ in $\mathcal{D}^{\prime}$. Define $F_{n}(x)=\int_{a}^{x} f_{n}$ and $F(x)=\int_{a}^{x} f$. If $g$ is a function of bounded variation and $F_{n} \rightarrow F$ as $n \rightarrow \infty$ uniformly on $[a, b]$, then $\int_{a}^{b} f_{n} g \rightarrow \int_{a}^{b} f g$ as $n \rightarrow \infty$.

\section{Main Results}

In this section, we firstly assume that $f$ satisfies the following assumptions:

( $\left.D_{1}\right) f(t, u)$ is $D_{H K}$-integrable with respect to $t$ for all $u \in C^{2}[0,1]$;

(D2) $f(t, u)$ is continuous with respect to $u$ for all $t \in[0,1]$, i.e. for each $t \in[0,1],\left\|f\left(t, u_{n}\right)-f(t, u)\right\| \rightarrow 0$ as $\left\|u_{n}-u\right\|_{C^{2}} \rightarrow 0$ for $u_{n} \in C^{2}[0,1]$;

$\left(D_{3}\right)$ There exist $f_{-}, f_{+} \in D_{H K}$ such that $f_{-}(\cdot) \leq f(\cdot, u) \leq f_{+}(\cdot)$ for all $u \in C^{2}[0,1]$.

Lemma 3.1 Assume that $1-2 \xi-2 \xi p \neq 0$. then, the NBVP is equivalent to the integral equation

$$
\begin{aligned}
u(t)= & c_{0}\left\{\int _ { 0 } ^ { 1 } \left((s-1)^{2}\left(t^{2}-2 \xi t-2 \xi p\right) f(s, u(s)) d s\right.\right. \\
& -\int_{0}^{\xi}(\xi-s)\left(2 t^{2}(1+p)-2 t-2 p\right) f(s, u(s)) d s \\
& \left.-\int_{0}^{t}(1-2 \xi-2 \xi p)(s-t)^{2} f(s, u(s)) d s\right\}, t \in[0,1],
\end{aligned}
$$

where $\xi$ is a constant with $0 \leq \xi \leq 1$ and $c_{0}=1 / 2(1-2 \xi-2 \xi p)$.

Proof. For all $t \in[0,1], s \in[0,1], u \in C[0,1], u^{\prime \prime} \in C[0,1]$, According to the lemma 2.5, we have

$$
\begin{aligned}
u^{\prime \prime}(t)-u^{\prime \prime}(0) & =-\int_{0}^{t} f(s, u(s)) d s, \\
u^{\prime}(t)-u^{\prime}(0) & =-\int_{0}^{t} \int_{0}^{s} f(\theta, u(\theta)) d \theta d s+u^{\prime \prime}(0) t \\
& =-\int_{0}^{t}(t-\theta) f(\theta, u(\theta)) d \theta+u^{\prime \prime}(0) t
\end{aligned}
$$


Then

$$
\begin{aligned}
u(t)-u(0) & =-\int_{0}^{t} \int_{0}^{s}(s-\theta) f(\theta, u(\theta)) d \theta d s+\frac{1}{2} u^{\prime \prime}(0) t^{2}+u^{\prime}(0) t \\
& =-\int_{0}^{t} \frac{1}{2}(t-s)^{2} f\left(s, u(s) d s+\frac{1}{2} u^{\prime \prime}(0) t^{2}+u^{\prime}(0) t .\right.
\end{aligned}
$$

According to the boundary conditions, we can get

$$
\left\{\begin{array}{l}
-\int_{0}^{1} \frac{1}{2}(s-1)^{2} f(s, u(s)) d s+\frac{1}{2} u^{\prime \prime}(0)+u^{\prime}(0)+u(0)=0 \\
-\int_{0}^{\xi}(\xi-s) f(s, u(s)) d s+u^{\prime \prime}(0) \xi+u^{\prime}(0)=0 \\
-p u^{\prime}(0)+u(0)=0
\end{array}\right.
$$

From (4) then, we can obtain

$$
\begin{aligned}
u^{\prime \prime}(0) & =\int_{0}^{1} \frac{(s-1)^{2}}{1-2 \xi-2 \xi p} f(s, u(s)) d s-\int_{0}^{\xi} \frac{2(1+p)(\xi-s)}{1-2 \xi-2 \xi p} f(s, u(s)) d s, \\
u^{\prime}(0) & =\int_{0}^{\xi} \frac{\xi-s}{1-2 \xi-2 \xi p} f(s, u(s)) d s-\int_{0}^{1} \frac{\xi(s-1)^{2}}{1-2 \xi-2 \xi p} f(s, u(s)) d s \\
u(0) & =\int_{0}^{\xi} \frac{p(\xi-s)}{1-2 \xi-2 \xi p} f(s, u(s)) d s-\int_{0}^{1} \frac{\xi p(s-1)^{2}}{1-2 \xi-2 \xi p} f(s, u(s)) d s .
\end{aligned}
$$

Furthermore from (3)and(4) we conclude that:

$$
\begin{aligned}
u(t)= & c_{0}\left\{\int _ { 0 } ^ { 1 } \left((s-1)^{2}\left(t^{2}-2 \xi t-2 \xi p\right) f(s, u(s)) d s\right.\right. \\
& -\int_{0}^{\xi}(\xi-s)\left(2 t^{2}(1+p)-2 t-2 p\right) f(s, u(s)) d s \\
& \left.-\int_{0}^{t}(1-2 \xi-2 \xi p)(s-t)^{2} f(s, u(s)) d s\right\}, t \in[0,1] .
\end{aligned}
$$

It is easy to calculate that NBVP (1) hold by taking the derivative both sides of (6). This completes the proof.

Lemma 3.2 (Theorem 6.15,[M. Schechter. (2004)]) The compact operator $\mathcal{A}: M \rightarrow M$ has at least one fixed point. When $M$ is bounded, closed, convex, nonempty subset of a Bananach space $X$ over $\mathbb{R}$.

With the help of the preceding lemmas, we can now prove the existence of solutions of the NBVP (1).

Theorem 3.1 Under the assumptions $\left(D_{1}\right)-\left(D_{3}\right)$ and $1-2 \xi-2 \xi p \neq 0$, then there exists at least one solution $u \in C^{2}[0,1]$ of the NBVP (1).

Proof. Let

$$
\begin{array}{lll}
F_{-}(t)=\int_{0}^{t} f_{-}(s) d s, & F_{+}(t)=\int_{0}^{t} f_{+}(s) d s, & F_{u}(t)=\int_{0}^{t} f(s, u(s)) d s ; \\
H_{-}(t)=\int_{0}^{t} F_{-}(s) d s, & H_{+}(t)=\int_{0}^{t} F_{+}(s) d s, & H_{u}(t)=\int_{0}^{t} F_{u}(s) d s .
\end{array}
$$

Since $f_{-}(s) \leq f(s, u(s)) \leq f_{+}(s)$ for all $u \in C^{2}[0,1]$, we can get

$$
\begin{gathered}
F_{-}(t) \leq F_{u}(t) \leq F_{+}(t), \quad H_{-}(t) \leq H_{u}(t) \leq H_{+}(t), \\
\int_{0}^{t} H_{-}(s) d s \leq \int_{0}^{t} H_{u}(s) d s \leq \int_{0}^{t} H_{+}(s) d s .
\end{gathered}
$$

Suppose that 


$$
\begin{gathered}
M_{1}=\max _{t \in[0,1]}\left|\int_{0}^{t} f_{-}(s) d s\right|+\max _{t \in[0,1]}\left|\int_{0}^{t} f_{+}(s) d s\right|, \quad M_{2}=\max _{t \in[0,1]}\left|\int_{0}^{t} F_{-}(s) d s\right|+\max _{t \in[0,1]}\left|\int_{0}^{t} F_{+}(s) d s\right| \\
M_{3}=\max _{t \in[0,1]}\left|\int_{0}^{t} H_{-}(s) d s\right|+\max _{t \in[0,1]}\left|\int_{0}^{t} H_{+}(s) d s\right|, \quad M_{4}=|u(0)|+2\left|u^{\prime}(0)\right|+\frac{5}{2}\left|u^{\prime \prime}(0)\right| .
\end{gathered}
$$

Then for each $t \in[0,1]$, and any $u \in C^{2}[0,1]$, we have

$$
\begin{gathered}
\left|F_{u}(t)\right| \leq \max _{0 \leq t \leq 1}\left|F_{-}(t)\right|+\max _{0 \leq t \leq 1}\left|F_{+}(t)\right|=M_{1}, \quad\left|H_{u}(t)\right| \leq \max _{0 \leq t \leq 1}\left|H_{-}(t)\right|+\max _{0 \leq t \leq 1}\left|H_{+}(t)\right|=M_{2}, \\
\left|\int_{0}^{t} H_{u}(s) d s\right| \leq \max _{0 \leq t \leq 1}\left|\int_{0}^{t} H_{-}(s) d s\right|+\max _{0 \leq t \leq 1}\left|\int_{0}^{t} H_{+}(s) d s\right|=M_{3} .
\end{gathered}
$$

Let $B=\left\{u \in C^{2}[0,1]:\|u\|_{C^{2}} \leq l, l=M_{1}+M_{2}+M_{3}+M_{4}>0\right\}$. For each $u \in B, t \in[0,1]$, define the operator

$$
\begin{aligned}
\mathcal{A} u(t)= & c_{0}\left\{\int _ { 0 } ^ { 1 } \left((s-1)^{2}\left(t^{2}-2 \xi t-2 \xi p\right) f(s, u(s)) d s\right.\right. \\
& -\int_{0}^{\xi}(\xi-s)\left(2 t^{2}(1+p)-2 t-2 p\right) f(s, u(s)) d s \\
& \left.-\int_{0}^{t}(1-2 \xi-2 \xi p)(s-t)^{2} f(s, u(s)) d s\right\} .
\end{aligned}
$$

Now we prove this theorem in three steps.

Step 1: $\mathcal{A}: B \rightarrow B$.

For all $u \in B$, by (3.7), one has

$$
\begin{aligned}
& \|\mathcal{A} u\|_{C^{2}}=\|u\|_{\infty}+\left\|u^{\prime}\right\|_{\infty}+\left\|u^{\prime \prime}\right\|_{\infty} \\
= & \max _{t \in[0,1]}\left|u(0)+u^{\prime}(0) t+\frac{1}{2} u^{\prime \prime}(0) t^{2}-\int_{0}^{t} \frac{1}{2}(t-s)^{2} f(s, u(s)) d s\right|+\max _{t \in[0,1]}\left|u^{\prime}(0)+u^{\prime \prime}(0) t-\int_{0}^{t}(t-s) f(s, u(s)) d s\right| \\
& +\max _{t \in[0,1]}\left|u^{\prime \prime}(0)-\int_{0}^{t} f(s, u(s)) d s\right| \\
\leq & \left(|u(0)|+2\left|u^{\prime}(0)\right|+\frac{5}{2}\left|u^{\prime \prime}(0)\right|\right)+\max _{t \in[0,1]}\left|\int_{0}^{t} \frac{1}{2}(t-s)^{2} f(s, u(s)) d s\right|+\max _{t \in[0,1]}\left|\int_{0}^{t}(t-s) f(s, u(s)) d s\right|+\max _{t \in[0,1]}\left|\int_{0}^{t} f(s, u(s)) d s\right| .
\end{aligned}
$$

For all $t \in[0,1]$, one has

$$
\begin{aligned}
& \max _{t \in[0,1]}\left|\int_{0}^{t} \frac{1}{2}(t-s)^{2} f(s, u(s)) d s\right|=\max _{t \in[0,1]}\left|\int_{0}^{t} \frac{1}{2}(t-s)^{2} d F_{u}(s) d s\right| \\
= & \max _{t \in[0,1]}\left|\frac{1}{2}(t-s)^{2} F_{u}(s)\right|_{s=0}^{s=t}+\int_{0}^{t}(t-s) F_{u}(s) d s\left|=\max _{t \in[0,1]}\right| \int_{0}^{t}(t-s) d H_{u}(s) \mid \\
= & \max _{t \in[0,1]}\left|(t-s) H_{u}(s)\right|_{s=0}^{s=t}-\int_{0}^{t} H_{u}(s) d s\left|=\max _{t \in[0,1]}\right| \int_{0}^{t} H_{u}(s) d s \mid \\
\leq & M_{3} .
\end{aligned}
$$

With the same argument, we have

$$
\begin{aligned}
& \max _{t \in[0,1]}\left|\int_{0}^{t}(t-s) f(s, u(s)) d s\right| \leq M_{2}, \\
& \max _{t \in[0,1]}\left|\int_{0}^{t} f(s, u(s)) d s\right| \leq M_{1} .
\end{aligned}
$$

In view of (9), (10), one has

$$
\|\mathcal{A} u\|_{C^{2}} \leq M_{4}+M_{3}+M_{2}+M_{1}
$$


Then $\|\mathcal{A} u\|_{C^{2}} \leq M_{4}+M_{3}+M_{2}+M_{1}=l$. Hence, $\mathcal{A}(B) \subseteq B$.

Step 2: $\mathcal{A}(B)$ is equi-continuous.

Let $t_{1}, t_{2} \in[0,1], u \in B$

$$
\begin{aligned}
\left|\mathcal{A} u\left(t_{1}\right)-\mathcal{A} u\left(t_{2}\right)\right|= & \mid u^{\prime}(0)\left(t_{1}-t_{2}\right)+\frac{1}{2} u^{\prime \prime}(0)\left(t_{1}^{2}-t_{2}^{2}\right)-\int_{0}^{t_{1}} \frac{1}{2}\left(t_{1}-s\right)^{2} f(s, u(s)) d s \\
& +\int_{0}^{t_{2}} \frac{1}{2}\left(t_{2}-s\right)^{2} f(s, u(s)) d s \mid \\
\leq & \left|t_{1}-t_{2}\right|\left(\left|u^{\prime}(0)\right|+\left|u^{\prime \prime}(0)\right|+\frac{1}{2}\left|t_{1}-t_{2}\right|\left|\int_{0}^{t_{1}}\left(t_{1}+t_{2}-2 s\right) f(s, u(s)) d s\right|\right. \\
& +\left|\int_{t_{1}}^{t_{2}} \frac{1}{2}\left(t_{2}-s\right)^{2} f(s, u(s)) d s\right| ; \\
\left|\mathcal{A} u^{\prime}\left(t_{1}\right)-\mathcal{A} u^{\prime}\left(t_{2}\right)\right|= & \left|u^{\prime \prime}(0)\left(t_{1}-t_{2}\right)-\int_{0}^{t_{1}}\left(t_{1}-s\right) f(s, u(s)) d s+\int_{0}^{t_{2}}\left(t_{1}-s\right) f(s, u(s)) d s\right| \\
\leq & \left|t_{1}-t_{2}\right|\left(\left|u^{\prime \prime}(0)\right|+\left|\int_{0}^{t_{1}} f(s, u(s)) d s\right|\right)+\left|\int_{t_{1}}^{t_{2}}\left(t_{2}-s\right) f(s, u(s)) d s\right| \\
\left|\mathcal{A} u^{\prime \prime}\left(t_{1}\right)-\mathcal{A} u^{\prime \prime}\left(t_{2}\right)\right| & \left|-\int_{0}^{t_{1}} f(s, u(s)) d s+\int_{0}^{t_{2}} f(s, u(s)) d s\right| \leq\left|\int_{t_{1}}^{t_{2}} f(s, u(s)) d s\right| .
\end{aligned}
$$

From step 1, we have drew the conclusion that $\left|\int_{0}^{t_{1}}\left(t_{1}+t_{2}-2 s\right) f(s, u(s)) d s\right|,\left|\int_{0}^{t_{1}} f(s, u(s)) d s\right|$ are bounded. Moreover, $\int_{t_{1}}^{t_{2}} \frac{1}{2}\left(t_{2}-s\right)^{2} f(s, u(s)) d s, \int_{t_{1}}^{t_{2}}\left(t_{2}-s\right) f(s, u(s)) d s, \int_{t_{1}}^{t_{2}} f(s, u(s)) d s$ are continuous on [0,1], hence uniformly continuous on $[0,1]$.

Then, from (11), (12), (13) we can conclude that $\mathcal{A}(B)$ is equi-continuous on[0,1] for all $u \in B$.

In view of step 1 , step 2 and Ascoli-Arzelà theorem, $\mathcal{A}(B)$ is relatively compact.

Step 3: $\mathcal{A}$ is a continuous mapping.

Let $u \in B,\left\{u_{n}\right\}_{n \in \mathbb{N}}$ be a sequence in $B$ and $u_{n} \rightarrow u$ as $n \rightarrow \infty$.

By $\left(D_{2}\right)$, one has

$$
f\left(\cdot, u_{n}\right) \rightarrow f(\cdot, u) \quad \text { as } \quad n \rightarrow \infty .
$$

According to the assumption $\left(D_{3}\right)$ and Lemma 2.4, we have

$$
\lim _{n \rightarrow \infty} \int_{0}^{t} f\left(s, u_{n}(s)\right) d s=\int_{0}^{t} f(s, u(s)) d s, \quad t \in[0,1] .
$$

It is easy to verify, by Lemma 2.6, that

$$
\lim _{n \rightarrow \infty} \mathcal{A}\left(u_{n}\right)=\mathcal{A}(u)
$$

Hence, $\mathcal{A}$ is continuous.

Thus, $\mathcal{A}$ satisfies the hypotheses of Lemma 3.2, then there exists a fixed point of $\mathcal{A}$ which is a solution of (2). By Lemma 3.1 , the NBVP (1) has at least one solution.

\section{Example}

In this section, we give an example for the application of Theorem 3.1.

Consider the boundary value problem

$$
\left\{\begin{array}{l}
u^{\prime \prime \prime}(t)=-t \cos u-r, \quad t \in[0,1] \\
u^{\prime}(\xi)=0 \\
u(0)-p u^{\prime}(0)=0, \\
u(1)=0 .
\end{array}\right.
$$


where $r$ is the distributional derivative of the Weiertrass function $R(t)=\sum_{n=0}^{\infty} a^{n} \sin b^{n} t$, and $0<a<1<b, a b>1$ in [G. H. Hardy. (1916)]. It is easy to see that $R(t) \in C[0,1]$ and $R(0)=0$, hence $r$ is $D_{H K}$-integrable. Let $f(t, u(t))=t \cos u+r$, then $f(t, u(t))$ satisfies $\left(D_{1}\right),\left(D_{2}\right)$. Moreover, let $f_{-}(t)=-t+r$ and $f_{+}(t)=t+r$, then

$$
f_{-}(\cdot) \leq f(\cdot, u) \leq f_{+}(\cdot),
$$

i.e., $\left(D_{3}\right)$ holds. Therefore, the initial value problem (14) has a solution.

Remark It is well known that the function $R(t)$ given by Riemann is continuous but pointwise differentiable nowhere on $[0,1]$, then the distributional derivative $r$ in (14) is neither $H K$ integrable nor Lebesgue integrable. Hence, this example is not covered by any result using HK integral or Lebesgue integral. Thus, Theorem 3.1 is more extensive.In this case, we can study more about the existence of the extended solutions of three-point nonlinear boundary-value problems and its application.

\section{References}

Anderson, D., Anderson, T., \& Kleber, M. (2006). Green's function existence of solutions for a functional focal differential equation. Electron. J. of differential Equations, 12, 1-14.

Ang, D. D., Schmitt, K., \& Vy, L. K. (1997). A multidimensional analogue of the Denjoy-Perron-Henstock-Kurzweil integral. Bull. Belg. Math. Soc., 4, 355-371.

Bugajewska, D. (1998). On the equation of $n$th order and the Denjoy integral. Nonlinear Anal., 34, 1111-1115. http://dx.doi.org/10.1016/S0362-546X(98)00035-2

Bullen, P. S., \& Sarkhel, D. N. (1987). On the solution of $\left(\frac{d y}{d x}\right)_{a p}=f(x, y)$. J. Math. Anal. Appl., 127, 365-376. http://dx.doi.org/10.1016/0022-247X(87)90115-6

Chew, T. S., \& Flordeliza, F. (1991). On $x^{\prime}=f(t, x)$ and Henstock-Kurzweil integrals. Differential Integral Equations, 4, 861-868.

Hardy, G. H. (1916). Weierstrass's Non-Differential Function. Trans. Amer. Math. Soc., 3, 301-325.

Lee, P. Y. (1989). Lanzhou Lectures on Henstock integration. World Scientific, Singapore.

Liang, B., \& Ye, G. (2015). On second order nonlinear boundary value problems and the distributional HenstockKurzweil integral,Boundary Value Problems. 73. http://dx.doi.org/10.1186/s13661-015-0338-0

Minghe, P., \& Chang, S. K. (2007). Existence and uniqueness of solutions for third-order nonlinear boundary value problems. J. Math, Anal. Appl., 327, 23-35. http://dx.doi.org/10.1016/j.jmaa.2006.03.057

Kurzweil, J. (1999). Henstock-Kurzweil integration:Its relation to topological vector spaces. World Scientific,Singapore.

Schechter, M. (2004). An Introduction to Nonlinear Analysis. Cambridge Univ. Press, Cambridge.

Schwabik, Š., \& Ye, G. (2005). Topics in Banach space integration. World Scientific, Singapore.

Talvila, E. (1999-2000). Limits and Henstock integrals of products. Real Anal. Exchange, 25, 907-918.

Talvila, E. (2008). The distributional Denjoy integral. Real Anal. Exchange, 33, 51-82.

Zhou, X., \& Ye, G. (2012). Second order periodic boundary value problems involving the distributional HenstockKurzweil integral. Advances in Pure Mathematics, 2, 330-336. http://dx.doi.org/10.4236/apm.2012.25046

\section{Copyrights}

Copyright for this article is retained by the author(s), with first publication rights granted to the journal.

This is an open-access article distributed under the terms and conditions of the Creative Commons Attribution license (http://creativecommons.org/licenses/by/3.0/). 